VIRAL INFECTIONS

\title{
Waking up the immune system
}

Inactivation of antiviral $\mathrm{T}$ cells is a key obstacle preventing the clearance of persistent infections by a wide range of viruses, including HIV, hepatitis $B$ virus (HBV) and hepatitis $C$ virus (HCV) in humans, and lymphocytic choriomenigitis virus (LCMV) in rodents. However, the mechanisms that initially induce immunosuppression and lead to viral persistence are unclear. Now, two papers report that interleukin-10 (IL-10) has an important role in this process, and provide evidence that IL-10 blockade might have therapeutic potential for chronic viral infections.

To determine the mechanism by which viruses induce immunosuppression, both Brooks and colleagues writing in Nature Medicine, and Ejrnaes and colleagues writing in the Journal of Experimental Medicine, used mice infected with LCMV clone 13 (LCMV13), which results in a chronic infection associated with impairment and deletion of virus-specific $\mathrm{CD} 8^{+} \mathrm{T}$ cells.
Brooks and colleagues observed increased IL-10 RNA in the spleens of LCMV13-infected mice, and the increased IL-10 production potentiated T-cell inactivation. However, in IL-10-deficient LCMV13-infected mice, there were increased numbers of virus-specific $\mathrm{T}$ cells, and no infectious virus could be isolated 9 or 42 days following infection. No increased immunopathology, nor increased levels of neutralizing antibodies were associated with the rapid viral clearance in these mice.

IL-10-deficient mice previously infected with LCMV13 were also resistant to re-infection after viral rechallenge, indicating the development of competent memory T-cell responses. Furthermore, antibody blockade of IL-10 after the establishment of infection increased numbers of virus-specific T cells and sustained T-cell activity, decreased viral titres, and in one case resulted in clearance of the virus.

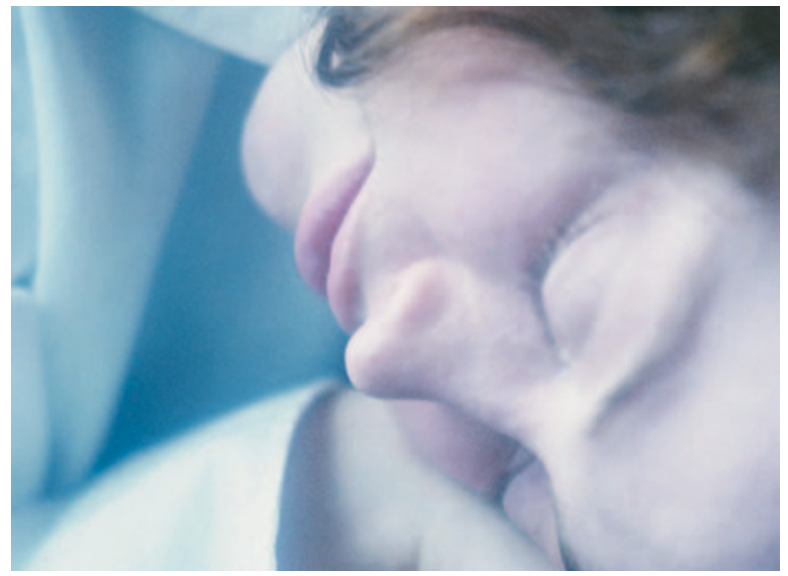

Ejrnaes and colleagues also showed that infection with LCMV13 resulted in increased IL-10 production in splenocytes, and suggested that the immune suppression mediated by LCMV13 was caused by a shift from antiviral interferon- $\gamma$ (IFN $\gamma$ ) production to the secretion of immunosuppressive IL-10.

The use of an antibody against IL-10 in LCMV13-infected mice resulted in low LCMV genomic-copy numbers and was effective during established infection, when it was able to reverse lymphopaenia. AntiIL-10 treatment resulted in a potent

\section{Targeting lung DCs to treat asthma}

Lung dendritic cells (DCs) are known to have a crucial role in the development of allergic asthma, through their activation of $\mathrm{T}$ helper $2\left(\mathrm{~T}_{\mathrm{H}} 2\right)$-cell responses. Therefore, strategies to interfere with their function might be a novel therapeutic approach for the treatment of asthma. In this study, Lambrecht and colleagues show that local treatment with the immunomodulator FTY720 suppresses experimentally induced asthma through the alteration of lung DC function.

FTY720 is a sphingosine-1-phosphate receptor agonist and is thought to act as an immunosuppressive agent by retaining lymphocytes in the lymphoid organs, thereby inhibiting lymphocyte influx into sites of inflammation. However, systemic treatment with this agent can result in peripheral lymphopaenia. So, the

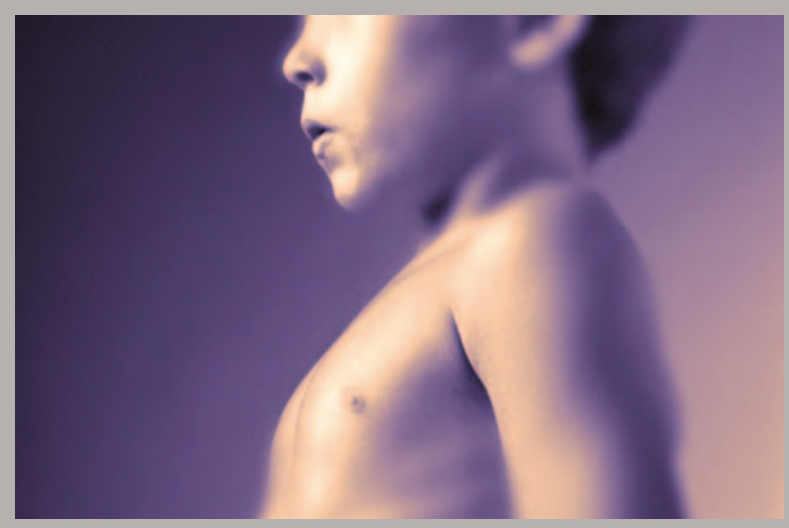

authors examined the effect of local administration of FTY720 in a mouse model of asthma.

Intratracheal administration of FTY720

before or during allergen challenge of sensitized mice suppressed the cardinal features of asthma, including eosinophilic inflammation, $\mathrm{T}_{\mathrm{H}}$ 2-type cytokine production and bronchial hyper-reactivity to a nonspecific stimulus. Notably, the number of lymphocytes in the peripheral blood and lymph nodes was not affected, indicating that lymphopaenia was not induced.

But if FTY720 does not sequester lymphocytes in lymphoid organs, how does it suppress the induction of asthma? The authors examined the effect of local administration of FTY720 on DC migration and found that treatment with FTY720 followed by allergen challenge did not affect the number of DCs in the periphery. However, the number of DCs in the draining lymph nodes of the lung was reduced, whereas the number of DCs detected in the lungs was greatly increased. Therefore, the data indicate that FTY720 prevents the migration of lung DCs to the draining lymph nodes, where they induce allergen-specific $\mathrm{T}_{\mathrm{H}}$ 2-cell responses.

Further examination of the effects of FTY720 on DC function in vitro showed that treatment of DCs with FTY720 
antiviral IFN $\gamma$-secreting $\mathrm{CD}^{+}{ }^{+} \mathrm{T}$-cell response, and decreased expression of programmed death 1 (PD1) on $\mathrm{T}$ cells, which has recently been identified as having a key role in sustaining suppression of $\mathrm{CD}^{+}$ T cells during persistent infection.

Elevated levels of IL-10 are observed during human HIV, HBV and HCV infections, and correlate with reduced T-cell activity and failure to control viral replication. Therefore, these two papers raise the possibility that IL-10 blockade might enhance the treatment of these persistent infections. And importantly, given that such a strategy is targeting a host factor that does not directly interact with the virus, it is unlikely that resistance owing to viral mutation would emerge, which is a cause of many treatment failures with current drugs.

Charlotte Harrison

ORIGINAL RESEARCH PAPERS Brooks, D. G. et al. Interleukin-10 determines viral clearance or persistance in vivo. Nature Med. 12, 1301-1309 (2006) | Ejrnaes, M. et al. Resolution of chronic viral infection after interleukin-10 blockade. J. Exp. Med. 203, 2461-2472 (2006) FURTHER READING Klenerman, P. \& Ludewig, B. Virus scores a perfect 10. Nature Med. 12 1246-1248 (2006)

directly inhibited the capacity of these cells to activate and polarize antigen-specific T cells. However, the maturation status of the DCs was not affected. The authors showed that the capacity of DCs to form stable and long-lasting interactions with T cells was abolished in the presence of FTY720, which might account for the inability of FTY720-treated DCs to stimulate T cells.

Therefore, FTY720 suppresses experimental asthma by blocking the migration of DCs from the lung to the draining lymph node without inducing peripheral lymphopaenia and by reducing the capacity of DCs to activate naive and effector $\mathrm{T}_{\mathrm{H}} 2$ cells. This study indicates that targeting lung DCs might be an effective strategy in the treatment of asthma.

Olive Leavy, Nature Reviews Immunology

ORIGINAL RESEARCH PAPER Idzko, M. et al. Local application of FTY720 to the lung abrogates experimental asthma by altering dendritic cell function.J. Clin. Invest. 116, 2935-2944 (2006)

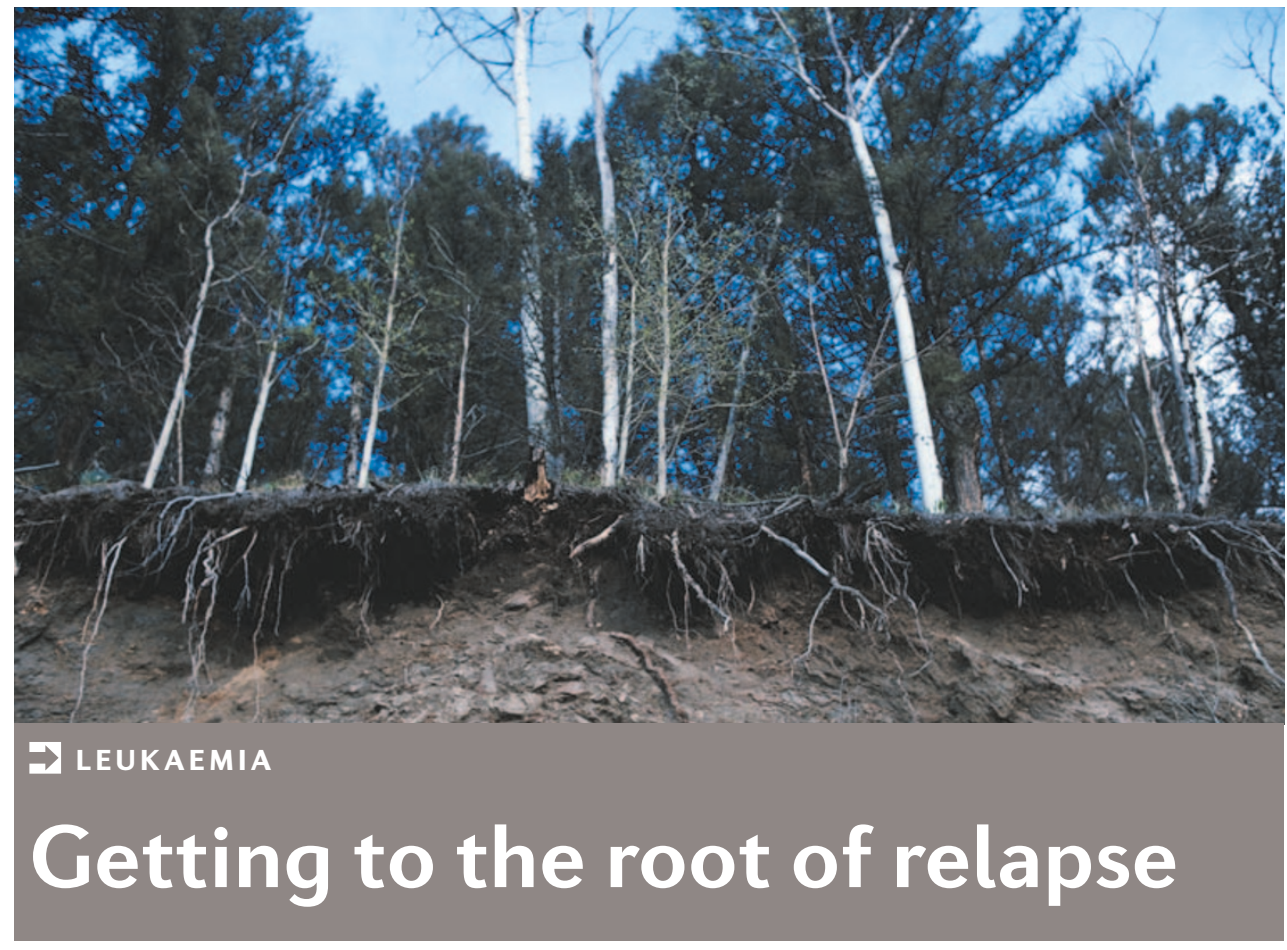

Patients with acute myeloid leukaemia (AML) currently face a bleak prognosis. Although chemotherapeutics targeting rapidly dividing tumour cells usually induce temporary remission, a small number of quiescent or slowly dividing leukaemic stem cells (LSCs) provide the seeds for relapse. Now, a study by Liquing Jin et al. raises hope that a tool targeting these elusive cells could be on the horizon. Reporting in Nature Medicine, the authors show that a new antibody treatment can interfere with the anchoring of LSCs in their hiding places and prompt them to differentiate, causing them to lose their cancerrepopulating capacity.

The concept of cancer stem cells has fundamentally changed the understanding of cancer development. Although cancer stem cells have only been identified for certain types of cancer, considerable research efforts are presently focusing on targeting these cells in the hope of achieving curative treatment for cancers that are prone to relapse.

In their study, Jin and colleagues discovered that the stem cells of AML can be manipulated with the activating monoclonal antibody $\mathrm{H} 90$, directed at the adhesion molecule CD44. In a murine model system of human AML, non-obese diabetic/severe combined immunodeficiency (NOD/SCID) mice were transplanted with human AML blast cells. The rare sub-population of LSCs in the human AML samples is characterized by the surface molecule signature $\mathrm{CD} 34^{+} \mathrm{CD} 38^{-}$, and is further defined by their capacity to initiate AML upon 'serial transplantation' of leukaemic cells from mouse to mouse. When NOD/SCID mice were treated with $\mathrm{H} 90$ a few weeks after AML transplantation, the leukaemic cell population was markedly reduced, and serially transplanted mice did not develop leukaemia. Closer analysis demonstrated that $\mathrm{H} 90$ directly targets CD34+CD38- LSCs, and interrupts their transport to stem-cell supportive microenvironmental niches in the bone marrow and spleen by reducing trans-endothelial cell migration, the last step in stem-cell homing. Moreover, CD44 ligation with the $\mathrm{H} 90$ antibody also directly enhanced the differentiation of LSCs into granulocytes and monocytes.

These findings identify CD44 as a key regulator of leukaemogeneisis (indeed, the expression of certain CD44 isoforms on AML cells is indicative of a poor prognosis), and demonstrates the dependence of LSCs on a micro-environmental niche, a finding which might provide an 'Achilles heel' that can be targeted to eradicate these cells. Furthermore, it has been shown that certain types of solid tumours are also maintained by a small number of cancer stem cells, and the authors speculate that CD44 might be involved here as well. So, their study provides hope for a potential generalized therapeutic strategy to eradicate cancer stem cells through interrupting their interaction with supportive micro-environments, and closer analysis could uncover further drug targets involved in this interaction.

Alexandra Flemming

ORIGINAL RESEARCH PAPER Jin, L. et al. Targeting of CD44 eradicates acute myeloid leukemic stem cells. Nature Med. 12, 1167-1174 (2006) 